\title{
A comparison of mono and multivalent linkers and their effect on the colloidal stability of nanoparticle and immunoassays performance.
}

\author{
Vladimir Gubala ${ }^{1 *}$, Xavier Le Guevel ${ }^{1}$, Rob Nooney ${ }^{1}$, David E. Williams ${ }^{2}$ and Brian MacCraith ${ }^{1}$ \\ ${ }^{1}$ Biomedical Diagnostics Institute, Dublin City University, Glasnevin, Dublin 9, Ireland \\ ${ }^{2}$ Mac Diarmid Institute for Advanced Materials and Nanotechnology, Department of Chemistry, University \\ of Auckland, Private Bag 92019, Auckland 1142, New Zealand
}

\begin{abstract}
When designing devices for biomedical diagnostics, increasing the signal to noise ratio is often critical for achieving clinically relevant sensitivity and limits of detection (LOD). In antibodybased assays, the measured signal can be amplified through the replacement of molecular fluorophores with doped nanoparticles (NP). However, the benefits of using NPs can only be realized if the NPs are coated efficiently with detection antibody, have good colloidal stability and the ratio of specific to non-specific binding (NSB) is high enough. The main focus of this paper is on the optimization of the bioconjugation protocol for antibody labeling of NPs leading to improved assay performance. Two types of linkers were used: monovalent linkers (glutaraldehyde; sulfo-SMCC; and sulfo-SIAB), and three generations of dendrimers endowed with multivalent carboxylic functionality. Overall, the NP-IgG conjugates prepared using multivalent linkers showed a significantly lower LOD and higher sensitivity than their homo- or hetero-functional counterparts. The multivalent dendrimers also improved NP stability and reduced aggregation. Moreover, the dendrimers showed a higher reactivity with biological material, a feature that could significantly reduce the cost of high-throughput biodiagnostics tests.
\end{abstract}

\section{Introduction:}

Fluorescent labels are used for a range of applications including immunosorbent assays[1, 2], immunocytochemistry[3-5], flow cytometry[6, 7] and DNA/protein microarray analysis[8, 9]. Fluorescence is preferred because it combines high sensitivity with a low limit of detection (LOD)[10-12]. Current research in biomedical diagnostics is moving to inexpensive devices, using biochips that require small samples volumes with minimum sample manipulation and high reliability under various experimental conditions. To meet this new demand, fluorescent labels with improved physical and chemical properties are required: for example, high photostability and fluorescence intensity, with a reproducible signal under a variety of chemical and biological 
conditions. Dye-doped silica nanoparticles[13-15] (NP) stand out as excellent candidates as it is possible to dope silica NPs with a large number of fluorophores, increasing the total fluorescence of the label significantly[16, 17]. Moreover, the fluorophore is protected inside a silica matrix, thereby increasing photostability[18, 19] and quantum efficiency[20, 21]. Silica NPs are also relatively non-toxic, chemically inert, and can be prepared in a range of sizes[22]. It also relatively easy to functionalize silica NP with bioreactive groups that enable facile bioconjugation[16, 22, 23].

We have found inconsistencies amongst bioassay data obtained using the same silica NP label with the same capture antibody but prepared using different bioconjugation protocols. Moreover, each step in the conjugation process modifies the zeta potential of the NP, thus affecting colloidal stability. We believe this is a significant stumbling block in the development of silica NP labels. In 2006, Tan and colleagues[16] proposed a way of improving colloidal stability of silica NPs through the addition of a negatively charged non-reactive organosilane alongside the bioreactive organosilane. In this paper we extend this work and show the significance, both for colloid stability and for assay performance, of the choice of the bi-linker, used to conjugate the detection antibody to the functionalised NP. There are many commercially available bi-linkers, for example glutaraldehyde[7], adipic acid[24], and succinic anhydride[22, 25]. Using glutaraldehyde, the biomolecule of interest is immobilized directly, via Schiff base formation. In many other cases additional chemicals are required for activation.

While a lot of work has been published on the functionalization of silica NP with a reactive organosilane[16, 26], to our best knowledge, no systematic studies have been reported for the optimization of the linker chemistry. There is a great choice of commercial hetero- or homo-functional linkers available; however, their effect on NP stability, aggregation, solubility and efficiency of bioconjugation is poorly documented. This is striking, particularly when considering that this is the actual layer "sensed" by the molecule to be labeled. Furthermore, in a sensitive and functional bioassay, a NP labeled biomolecule must retain its specific binding to analyte, while keeping the non-specific adsorption (NSA) on the substrate to a minimum. Therefore, the focus of this work is to investigate the effect of the linker on NP stability and the performance of NP labeled molecules in a sandwich immunoassay. In particular, the LOD and sensitivity was determined for the detection of human IgG.

For this study, three monovalent bilinkers (Glutaraldehyde, Sulfosuccinimidyl 4-[Nmaleimidomethyl]cyclohexane-1-carboxylate (sulfo-SMCC) and N-Sulfosuccinimidyl[4iodoacetyl]aminobenzoate (sulfo-SIAB)), and three multivalent linker molecules (dendrimers, generations 0,1 and 2) were selected for the bioconjugation of NPs with antibodies. Multivalent 
dendrimers are attracting a great deal of interest for applications in biomedical diagnostics[2730], pharmacology / drug delivery [31, 32] [33], cancer research[34], and nanomedicine[35]. Their use in research is still unabated now, 30 years after their first reported synthesis by Vogtle[36]. Dendrimers are branched, structurally appealing, monodispersed compounds with a well-defined structure, in which the number and functionality of the functional groups can be tailored for the specific bioconjugation required. In this work we show that the multivalency of dendrimers leads to a higher efficiency in bioconjugation of detection antibodies and an improved assay performance. Such improvements are necessary for reducing costs in medical diagnostics, particularly where large numbers of samples are tested.

In this work we use near-infrared dye-doped silica NPs. At near infrared wavelengths there is low background interference from the fluorescence of biological molecules, solvent, and substrates. Furthermore, whole blood has a weak absorption in the NIR region, thus reducing the need for whole-blood filtering for assays using whole blood. NIR light can also penetrate skin and tissue to several millimeters and this can enable fluorescence detection in dermatological or invivo diagnostic devices.

The three bivalent linkers used in this study are shown in the top half of figure 1 . The structure and mechanism for attachment of the carboxylic acid terminated dendrimers is shown in the bottom half of figure 1. Nanoparticles were characterized using transmission electron microscopy and dynamic light scattering.

\section{Experimental Section}

\section{Materials.}

Triton ® X-100 (trademark Union Carbide), n-hexanol (anhydrous, >99\%), cyclohexane (anhydrous $99.5 \%$ ), ammonium hydroxide $\left(28 \%\right.$ in $\left.\mathrm{H}_{2} \mathrm{O}>99.99 \%\right)$, tetraethylorthosilica (TEOS, $99.99 \%$ ), aminopropyltrimethoxysilane (APTMS, 97 \%), aminopropyltriethoxysilane (APTES, 99 \%), 3-(trihydroxysilyl)propyl methyl phosphonate, monosodium salt solution (THPMP, 42 wt \% in water), absolute ethanol, monobasic sodium phosphate, dibasic sodium phosphate. phosphate buffered saline (PBS, pH 7.4, $0.01 \mathrm{M}$ ), Tween ${ }^{\circledR} 20$ (trademark Uniqema), glutaraldehyde (25 wt \% in water), sodium azide $(99.99 \%)$ and albumin from bovine serum (BSA, $98 \%$ ) were all purchased from Sigma Aldrich Ireland, sulfo-SMCC and sulfo-SIAB were purchased from Pierce Chemical Company, dendrimers generations 1 and 2 were purchased from Frontier Scientific UK and used without further purification. Dendrimer generation 0 was synthesized in two simple steps starting from cyanuric chloride as starting material and purified on silica gel chromatography column prior to use[37]. Black 96 well plates used in the 
immunoassay were purchased from AGB Scientific Ireland. Deionised water (< $18 \mathrm{M} \Omega$ ) was obtained from a Milli-Q system from Millipore Ireland.

The dye used in this work is 4,5-Benzo-1'-ethyl-3,3,3',3'-tetramethyl-1-(4sulfobutyl)indodicarbocyanin-5'-acetic acid N-succinimidyl ester, or more commonly referred to as NIR-664-N-succinimidyl ester (purchased from Sigma Aldrich). This dye has a quantum efficiency of $23 \%$, a molar absorptivity of $187,000 \mathrm{~L} \mathrm{~mol}^{-1} \mathrm{~cm}^{-1}$ and fluorescence excitation and emission wavelengths of $672 \mathrm{~nm}$ and $694 \mathrm{~nm}$, respectively, in isopropanol[28].

\section{Instrumentation}

TEM micrographs were obtained using a Hitachi 7000 Transmission Electron Microscope operated at $100 \mathrm{kV}$. Images were captured digitally using a Megaview 2 CCD camera. Specimens were prepared by dropping aqueous solutions of the NPs onto a formvar carbon coated copper grid. Fluorescence measurements were performed on a Safire (Tecan) microplate reader. For NIR-664-N-succinimidyl ester-doped NPs, the excitation and emission wavelengths were set at $672 \mathrm{~nm}$ and $700 \mathrm{~nm}$, respectively. Dynamic light scattering (DLS) measurements were performed on a Zetasizer from Malvern instruments to yield values of zeta potential $(\xi)$ for the NPs.

\section{Methods}

\section{Synthesis of silica NPs}

Firstly, $1.62 \mathrm{mg}$ NIR-664-N-succinimidyl ester was dissolved in $2 \mathrm{~mL}$ anhydrous $\mathrm{n}$-hexanol. To this solution, was added APTES in ten molar excess. We prepared NPs containing 2 wt \% NIR664-succinimidyl ester using the microemulsion method[34]. Briefly, the microemulsion was formed by mixing cyclohexane oil phase $(15 \mathrm{~mL}), n$-hexanol co-solvent $(1.6 \mathrm{~mL})$, Triton ${ }^{\circledR} \mathrm{X}$ 100 surfactant $(3.788 \mathrm{~g})$ and $2 \mathrm{~mL}$ of the dye conjugate in $30 \mathrm{~mL}$ plastic bottles. Following this, $0.2 \mathrm{~mL}$ of TEOS and $0.16 \mathrm{~mL}$ of $\mathrm{NH}_{4} \mathrm{OH}$ were added to start the growth of the silica NPs. The reaction was stirred for $24 \mathrm{hrs}$, after which $0.1 \mathrm{ml}$ TEOS was added with rapid stirring.After 30 minutes, $0.08 \mathrm{~mL}$ of the organosilane, 3-(trihydroxysilyl)propyl methyl phosphonate, monosodium salt solution (THPMP), (42 wt \% in water) was added with stirring to prevent aggregation of the nanoparticles[18]. After a further 5 minutes, $0.02 \mathrm{~mL}$ of bioreactive organosilane, aminopropyltrimethoxysilane, (APTMS) was added to and the solution stirred for a further 12 hours. The APTMS has a free primary amine group for crosslinking to biomolecules. The NPs were separated from the solution with the addition of excess absolute ethanol and centrifuged twice with ethanol and once with deionised water. Sonication was used between the 
washing steps to resuspend the NPs. The NPs were dispersed in deionised water, at $2.0 \mathrm{mg} / \mathrm{mL}$ and stored in the dark at $4^{\circ} \mathrm{C}$. The NPs were characterized by Dynamic Light Scattering, Fluorescence Spectrophotometer and Transmission Electron Microscopy (Fig. 2).

\section{Synthesis of dendrimer G0.}

Intermediate 1: To a pressure vessel containing $100 \mathrm{~mL}$ of $\mathrm{CH}_{2} \mathrm{Cl}_{2}: \mathrm{MeOH}$ (1:1), 2-Amino-2(hydroxymethyl)-1,3-propanediol (3.9 g, $33 \mathrm{mmol}$ ) and Hunig's base (8.4 g, $65 \mathrm{mmol}$ ) were added. The mixture was cooled in an ice bath before addition of cyanuric chloride ( $3 \mathrm{~g}, 16 \mathrm{mmol})$. The reaction was then allowed to warm up to room temperature and stirred for 24 hours before 4piperidine carboxylic acid $(8.4 \mathrm{~g}, 65.2 \mathrm{mmol})$ and $\mathrm{NH} 4 \mathrm{OH}(5 \mathrm{~mL})$ were added. The reaction mixture was then heated up to $60^{\circ} \mathrm{C}$ and stirred overnight. Upon cooling, the precipitates were filtered and the solvent was removed by evaporation. The residue was re-dissolved in water (20 $\mathrm{mL}$ ) and acidified to $\mathrm{pH}=4-5$. Precipitates were formed, filtered off and washed extensively with water to give off-white powder ( $3.44 \mathrm{~g}, 47 \%$ yield). This intermediate was used in the second step without further purification.

Dendrimer G0: Intermediate 1 (0.5 g, $1.1 \mathrm{mmol})$ and diglycolic anhydride (3g, $16 \mathrm{mmol})$ were placed in a sealed vessel and irradiated in a CEM microwave at $300 \mathrm{~W}, 120^{\circ} \mathrm{C}$ for 5 minutes. Upon cooling, precipitates were formed, the whole mixture was dissolved in water and the $\mathrm{pH}$ was adjusted to 4-5. The solution was concentrated and the residue was purified on silica gel chromatography column with $\mathrm{MeOH} / \mathrm{CH}_{2} \mathrm{Cl}_{2}$ (5:95) as an eluent to yield oily product (440 mg, $35 \%$ yield).

\section{Bioconjugation}

All bioconjugation reactions were performed with the NIR-NP at the NP concentration of $2 \mathrm{mg} / \mathrm{mL}$, cross-linker concentration of $4 \mathrm{mM}$ and with $268 \mu \mathrm{g}$ of goat anti-human $\mathrm{IgG}$. The conjugation protocols were optimized for each linker with respect to the nature of its reactive groups.

Glutaraldehyde ( $4 \mu \mathrm{mol})$ was added into a $1 \mathrm{~mL}$ solution of NIR-NP $(2 \mathrm{mg} / \mathrm{mL})$ and gently shaken for 1 hour. The excess of unreacted linker was removed by means of centrifugation $(3 x)$ and the precipitates were dissolved in $946 \mu \mathrm{L}$ of $0.1 \mathrm{M}$ PBS buffer, $\mathrm{pH}=7.4$. To this solution, $54 \mu \mathrm{L}$ (268 $\mu \mathrm{g}$ ) of goat anti-human IgG was added and the mixture was allowed to shake for 4 hours. The excess of the unreacted protein was removed by repeated centrifugation (4x) and the final bioconjugation product was dissolved in $1 \mathrm{~mL}$ of $0.1 \mathrm{M}$ PBS buffer, $\mathrm{pH}=7.4$. Sodium azide 
$\left(\mathrm{NaN}_{3}\right)$, as an anti-microbial agent was further added into the mixture so that its final concentration reached $0.01 \%$.

The bioconjugation reaction using the heterofunctional linkers comprised two steps. First, sulfoSIAB or sulfo-SMCC $(4 \mu \mathrm{mol})$ were allowed to react with $1 \mathrm{~mL}$ of a solution of NIR-NP $(2 \mathrm{mg} / \mathrm{mL})$ in $0.1 \mathrm{M}$ PBS buffer, $\mathrm{pH}=7.2$, containing $5 \mathrm{mM}$ of EDTA for 30 minutes, after which the excess of the linker was removed by centrifugation. Second, 2-Iminothiolane $\cdot \mathrm{HCl}(16.8 \mathrm{nmol}$, Traut's reagent) was reacted with goat anti-human $\operatorname{IgG}(268 \mu \mathrm{g}, 1.68 \mathrm{nmol})$ in $250 \mu \mathrm{L}$ of $0.1 \mathrm{M}$ Phosphate Buffered Saline (PBS) buffer, $\mathrm{pH}=8.0$, containing $5 \mathrm{mM}$ of EDTA for 1 hour, after which the excess of the unreacted iminothiolane was removed using a Zeba desalting column (Pierce Chemical comp.).

Both fractions were subsequently mixed together in a reaction vessel for 4 hours, while keeping the total volume of the solution to $1 \mathrm{~mL}$. The reaction mixture was then purified by repeated centrifugation (4x) and re-dissolved in $0.1 \mathrm{M}$ PBS buffer, $\mathrm{pH}=7.4,0.01 \% \mathrm{NaN}_{3}$.

The $-\mathrm{COOH}$ groups of all three generations of the dendrimers were first activated with EDC/NHS mixture in 0.1 M 2-(N-morpholino)ethanesulfonic acid (MES) buffer, $\mathrm{pH}=6.3$ before reacting with the protein. For example, G0 $(4 \mu \mathrm{mol}, 7 \mathrm{x}-\mathrm{COOH})$ was dissolved in $0.5 \mathrm{~mL}$ of MES buffer. To this solution, NHS (42 $\mu \mathrm{mol}, 1.5$ equiv. per one -COOH group) and EDC (168 $\mu \mathrm{mol}$, 6.0 equiv. per one $-\mathrm{COOH}$ group) were added, the final volume was adjusted to $1 \mathrm{~mL}$ with MES buffer and the reaction was allowed to proceed for 30 minutes. The NHS activated dendrimer was then directly added into the NIR-NP $(2 \mathrm{mg} / \mathrm{mL})$, while keeping the total volume to $1 \mathrm{~mL}$. This mixture was allowed to react for 30 minutes; subsequently the excess of solution-free dendrimer was removed by centrifugation. The dendritic-modified NIR-NP were re-dissolved in $946 \mu \mathrm{L}$ of MES buffer, $\mathrm{pH}=7.0$ and goat anti-human $\operatorname{IgG}(135 \mu \mathrm{g}, 54 \mu \mathrm{L}$ from stock) was added. The reaction was allowed to shake for 4 hours, then $100 \mu \mathrm{L}$ of sodium bicarbonate buffer at $\mathrm{pH}=9.0$ was added to hydrolyze the remaining NHS esters into carboxyls and the mixture was purified by centrifugation $(4 \mathrm{x})$. The NIR-NP - IgG bioconjugate was re-dissolved in $0.1 \mathrm{M}$ PBS buffer, $\mathrm{pH}=7.4$, with $0.01 \mathrm{w} / \mathrm{v} \% \mathrm{NaN}_{3}$ and $0.5 \mathrm{w} / \mathrm{v} \%$ of BSA.

All reactions in which NIR-NPs were used were performed under reduced light (reaction vessels wrapped up in aluminum foil) to prevent photo-bleaching and all final samples were stored in the dark at $4^{\circ} \mathrm{C}$ overnight before they were used in the immunoassay.

\section{Fluorescence linked immunosorbent assay (FLISA)}


A sandwich assay format was used to test the performance of the NPs conjugated to human IgG through the linkers shown in figure 1 . In the first step $100 \mu \mathrm{L}$ of polyclonal goat-anti human $\mathrm{IgG}$, at $5 \mu \mathrm{g} / \mathrm{mL}$ was added to each well of a standard 96 well microplate. The plate was then incubated overnight at $4^{\circ} \mathrm{C}$. To remove any non-adsorbed antibody the plate was rinsed three times with PBS and three times with PBS/0.05\% w/v Tween ${ }^{\circledR}$. To prevent non-specific adsorption, $200 \mu \mathrm{L}$ of $1 \%$ w/v Bovine Serum Albumine (BSA) in PBS was added to each well and the plate incubated at $37{ }^{\circ} \mathrm{C}$ for 1 hour. The rinse cycle was then repeated. Following this, $100 \mu \mathrm{L}$ aliquots of human IgG in $0.1 \%$ w/v BSA were added in a series of dilutions from 10,000 $\mathrm{ng} / \mathrm{mL}$ to $0.01 \mathrm{ng} / \mathrm{mL}$ to each well and the plate incubated at $37^{\circ} \mathrm{C}$ for 1 hour. The rinse cycle was repeated to remove any non-specifically bound human IgG. Finally, $100 \mu \mathrm{L}$ aliquots of polyclonal goat anti-human IgG conjugated NPs at optimized value of $0.2 \mathrm{mg} / \mathrm{mL}$ (determined by an assay performed at saturated levels of both the captured antibody $(50 \mu \mathrm{g} / \mathrm{mL})$ and the antigen $(10,000 \mathrm{ng} / \mathrm{mL})$ by varying the concentration of the detection antibody) were added to each well and the plate incubated for a further hour at $37{ }^{\circ} \mathrm{C}$ in the dark. Prior to analysis the plate was rinsed one more time.

The fluorescence signal including standard deviation at each concentration of human $\operatorname{IgG}$ was determined from seven replicate experiments. The complete assay was fitted using a standard sigmoidal logistics fit (see below, left hand side) and the low concentrations of human IgG were fitted using an allometric power function (see below, right hand side)[38].

$$
F_{c}=\frac{F_{0}-F_{\max }}{1+\left(C / C_{o}\right)^{p}}+F_{\max } \quad F_{c}=F_{0}+k\left(\frac{C}{l}\right)^{m}
$$

where, Where, $F_{c}$ is the fluorescence at antigen concentration $C, F_{o}$ is the background fluorescence, $F_{\max }$ is the maximum fluorescence at the highest loading of antigen, $p$ is the power, and $C_{o}$ is the point of inflection. For the allometric function, $k$ and $m$ are the coefficient and power variables that define the gradient of the fit and $l$ normalizes the fit with antigen concentration.

The LOD of human $\operatorname{IgG}$ was defined as the concentration corresponding to a fluorescence signal 3 times the standard deviation of the background signal. The sensitivity of the assay (change in fluorescence signal with concentration of human $\mathrm{IgG}$ ) was determined at a range of concentrations through differentiation of the allometric power function. 


\section{Results and Discussion}

\section{Effect of the crosslinkers on colloidal stability}

Maintaining a high degree of monodispersity of the dye-doped nanoparticles is fundamental to successful bioassays. Current approaches to prepare colloidal solutions with low polydispersity index are mostly based on electrostatic interactions. The post-synthetic modification of the particle surface with charged species (e.g. phosphates, carboxylic acids etc.) is not only aimed to provide some sort of reactive handles but primarily to reduce the particle aggregation[39]. While the biomolecules of interest that are to be linked with the nanoparticles are usually charged species (proteins, ssDNA etc.) and have stabilizing effect on the bioconjugates[40], little attention was paid to the effect of the crosslinker used in the step preceding the reaction with the antibody. As seen in figure 3, monovalent linkers, glutaraldehyde and sulfo-SMCC had actually a destabilizing effect on the NP with the tendency to form aggregates. Sulfo-SMCC showed two different populations, the first transition corresponding to the formation of trimers or tetramers, with an average diameter of $275 \mathrm{~nm}$, and the second corresponding to larger aggregates with polydispersed characteristics. On the contrary, NPs modified with the multivalent dendrimers G1 and G2 showed only a small increase in particle diameter. The zeta potential increased from $-29.8 \mathrm{mV}$ to $-47.9 \mathrm{mV}$ and $-46.0 \mathrm{mV}$ for $\mathbf{G 1}$ and $\mathbf{G 2}$, respectively. The colloidal stability of dendrimer coated NPs is due to the significant increase in the $\zeta$ potential on addition of the linker. This is because the NHS ester activated groups on the dendrimer are susceptible to hydrolysis, which leads to the formation of carboxylic groups that are negatively charged at physiological $\mathrm{pH}$. Therefore, the electrostatic charge on the NP is increased and colloidal stability is maintained.

Immobilization of a model protein, Green Fluorescent Protein (GFP), on the modified NP surface seemed to have little effect on the zeta potential of the NP modified with the charged G1 and G2, while the changes in case of neutral Glu and sulfo-SMCC were more dramatic (figure 3B).

\section{Optimisation of bioconjugation}

As noted in the Experimental section, the bioconjugation of the GFP on the dendrimer-modified surface was performed via a well-known technique for the conjugation of carboxyl to amine groups in peptides and proteins. The reaction between carboxylic acids and amines to form stable amides is commonly facilitated by an addition of a carbodiimide, such as 1-Ethyl-3-[3dimethylaminopropyl] carbodiimide hydrochloride (EDC). However, EDC itself is not particularly efficient in crosslinking because in the first step it forms an unstable $\mathrm{O}$-acylisourea 
ester that is prone to fast hydrolysis and regeneration of the carboxyl. The carboxylic acids of the dendrimers were therefore activated in the presence of a mixture of N-hydroxysuccinimide (NHS) and a dehydrating agent, EDC. NHS/EDC mixture is commonly used to convert-COOH groups to semi-stable amine reactive NHS-esters. We realized that the availability and the lifetime of the NHS esters on the dendritic linker would determine the reaction efficiency and yields in the conjugation with the biomolecule that needs to be labeled. The activation reaction with EDC and NHS is most efficient at slightly acidic pH and usually performed in MES buffer at $\mathrm{pH} 4.7$ $6.0[41,42,27]$. With that in mind, we decided to explore on the effect of some other variables present in the EDC/NHS activation step such as the molar excess of NHS per COOH group, the ratio of the EDC/NHS co-reactants and the reaction time needed for the $\mathrm{COOH}$ activation. The optimum balance between the rate of aminolysis and the hydrolysis of the dendritic $\mathrm{N}$ succinimidyl esters was very important for maintaining high activity and specificity of the labeled antibody towards the antigen in the immunoassay. Green Fluorescence Protein (GFP) was used as a model protein in the conjugation to the dye-doped nanoparticles under various conditions. The coupling efficiency was then determined by means of fluorescence with the excitation maximum at $398 \mathrm{~nm}$ and emission maximum at $508 \mathrm{~nm}$, far from the fluorescence maxima of the NIR-NPs. After much experimenting with various parameters, the optimal conditions were found and read as follows: reaction medium - 0.1 M MES buffer, $\mathrm{pH}=6.0$; activation time -30 minutes; two fold molar excess of NHS reagent per COOH group and 4:1 ratio of EDC:NHS (Fig. 4).

\section{Concentration of the biomolecule}

Another very important parameter in the bioconjugation protocol is the concentration of the biomolecule that will comprise a monolayer on the NP surface. This amount is usually estimated and depends upon factors such as the molecular weight of the protein, its relative affinity for the particle and the particle size[43]. While the amount of protein required to achieve surface saturation can be calculated, the actual protein concentration in the reaction may be substantially higher than the "monolayer $=1$ equivalent of protein" value. The optimal value is usually given by the coupling efficiency, concentration of the target molecules and the amount of the non-specific binding and is usually determined experimentally. Therefore, we decided to investigate the effect of the concentration of the protein on the reaction yield. We compared the coupling efficiency of two linkers, sulfo-SMCC and G2, in the bioconjugation reaction using a model protein, green fluorescence protein (GFP). The reactions were carried out at two different concentrations of the GFP. First, the reaction was run with the amount of GFP representing 1.5 equivalent of the theoretical value necessary for surface saturation, and then a second reaction 
was performed using 3.0 equivalents. Figure 5 shows the effect of the increased concentration of GFP from 1.5 to 3.0 equivalents on the conjugation efficiency. The normalized fluorescence signal for sulfo-SMCC nearly doubled at the higher GFP concentration (3.0) when compared to the lower GFP concentration (1.5). Nevertheless, it was still significantly lower than the signal observed for the multivalent $\mathbf{G 2}$ linker, and that applied even for the case when the $\mathbf{G} \mathbf{2}$ sample contained only 1.5 equivalents of GFP. It is also noteworthy that the differences between the conjugation efficiency of the two $\mathbf{G 2}$ samples were very small. It suggests that the multivalency of the dendrimer is a significant factor responsible for the improvement in the reaction yields even at lower protein concentration. This is very important, particularly when considering the cost of the bioorganic material that is used in bioconjugation reactions.

\section{Fluorescence-Linked Immunosorbent Assay}

In order to test the performance of the dye doped silica NPs, we carried out a standard fluorescence-linked immunosorbent assay for the detection of human $\mathrm{IgG}$. We used a sandwich assay format, in which the capture and detection antibodies were polyclonal goat anti-human IgG. Seven different NP assays were performed using each of the mono- or multi- valent bilinkers. As seen in figure 6, all assays showed standard sigmoidal behavior, excluding NPs prepared using G2 dendrimer, where the signal did not saturate at high concentrations. This response is typical of multilayer adsorption and may be due to the poor solubility exhibited by larger dendrimeric structures. The normalized fluorescence ratio, at the highest concentration of human $\operatorname{IgG}$, $\left(F_{\max } / F_{o}\right)$ for multivalent NP labels was significantly greater than the fluorescence achieved from labels prepared using monovalent linkers (Figure 6 and table 1).

As seen in table 1, all the multivalent labels showed lower LOD than the monovalent linkers. Moreover, the LOD for G1 dendrimer was lower by two orders of magnitude than that for the popular and routinely used homofunctional linker glutaraldehyde. Therefore, NPs sensitized through the multivalent linkers are sensing lower concentrations of antigen and must be bound more efficiently to the antigen coated surface. From the work presented herein on the bioconjugation of GFP and other studies, it is likely the multivalent NPs are coated with several detection antibodies. This would increase the number of binding sites per label increasing the reactivity of the label. Furthermore, because the footprint of a single NP label is significantly larger than the diameter of antigen, it may be possible for two antigens to bind to a single NP at the same time. The LOD for the dendrimeric NPs compares favorably with published data on the 
detection of biotinylated hIgG using avidin labeled silica NPs, where an LOD of $1.9 \mathrm{ng} / \mathrm{mL}$ was observed[25].

For all three labels prepared using dendrimeric cross-linkers the assay showed good sensitivity at low concentrations of human IgG, with G1 showing the best response. We presume that the main reason why $\mathrm{G} 2$ dendrimer was outperformed by its smaller multivalent counterparts is due to its increased capacity to capture the protein at multiple locations, which can then restrict the binding capability of the labeled-antibody. Nonetheless, for NP labels prepared using the monovalent bilinker no sensitivity was observed at concentrations of human IgG below $1 \mathrm{ng} /$ $\mathrm{mL}$. At higher concentrations all samples showed good sensitivity.

\section{Conclusion}

Successful detection devices for biomedical diagnostics frequently require high sensitivity and low LOD. Moreover, a device for point-of-care diagnostics must be both inexpensive and reliable under a variety of experimental conditions. The data presented in this paper focused on finetuning the bioconjugation protocol in the labeling of biologically relevant material with NPs. We employed two types of linkers: monovalent linkers (glutaraldehyde; sulfo-SMCC; and sulfoSIAB), and three generations of dendrimers with multivalent carboxylic functionality. The NPs prepared using dendrimer generation 1 showed a significantly lower LOD and higher sensitivity than the homo- and hetero-functional crosslinkers. The difference in performance is remarkable, particularly when compared with glutaraldehyde, one of the most common crosslinker still employed by many research laboratories. We believe the multivalency of the dendrimers is one of the most significant factors behind the increase in the detection sensitivity. As revealed in this work, dendrimers have a positive effect on NP stability and aggregation. Moreover, they are more reactive with biological materials, capable of immobilizing antibody at the appropriate surface density while maintaining its activity to the analyte of interest. These properties are required for managing cost, in the design and implementation of biomedical devices, where expensive biological materials are necessary. Dendrimers are structurally appealing molecules that have already shown their high potential in the surface science of bioassay devices and this study confirmed that they are emerging as a powerful tool for the labeling of biomolecules in biomedical diagnostics as well.

\section{Aknowledgement}


This material is based upon works supported by the Science Foundation Ireland (S.F.I.) under Grant No. 05/CE3/B754.

\section{References}

[1] S. Nagl, M.I.J. Stich, M. Schaferling, O.S. Wolfbeis, Anal. Bioanal. Chem. 393 (2009) 1199-1207.

[2] C.S. Thaxton, D.G. Georganopoulou, C.A. Mirkin, Clin. Chim. Acta 363 (2006) $120-126$.

[3] R.S. Davidson, M.M. Hilchenbach, Photochem. Photobiol. 52 (1990) 431-438.

[4] M.L.R. Lim, M.G. Lum, T.M. Hansen, X. Roucou, P. Nagley, J. Biomed. Sci. 9 (2002) 488-506.

[5] R.S. Davidson, M.M. Hilchenbach, THE USE OF FLUORESCENT-PROBES IN IMMUNOCHEMISTRY, Bologna, Italy, 1989, pp. 431-438.

[6] M. Lowe, A. Spiro, Y.Z. Zhang, R. Getts, Cytometry A 60A (2004) 135-144.

[7] H.H. Yang, H.Y. Qu, P. Lin, S.H. Li, M.T. Ding, J.G. Xu, Analyst 128 (2003) 462-466.

[8] M. Takahashi, K. Nokihara, H. Mihara, Chem.Biol. 10 (2003) 53-60.

[9] H. Binder, T. Kirsten, I.L. Hofacker, P.F. Stadler, M. Loeffler, J. Phys. Chem. B 108 (2004) 18015-18025.

[10] X. Hun, Z.J. Zhang, Biosens. Bioelectron. 22 (2007) 2743-2748.

[11] A. Sukhanova, M. Devy, L. Venteo, H. Kaplan, M. Artemyev, V. Oleinikov, D. Klinov, M. Pluot, J.H.M. Cohen, I. Nabiev, Anal. Biochem. 324 (2004) 60-67.

[12] Q. Zhang, L.H. Guo, Bioconjugate Chem. 18 (2007) 1668-1672.

[13] J.L. Yan, M.C. Estevez, J.E. Smith, K.M. Wang, X.X. He, L. Wang, W.H. Tan, Nano Today 2 (2007) 44-50.

[14] A. Burns, H. Ow, U. Wiesner, Chem. Soc. Rev. 35 (2006) 1028-1042.

[15] C.E. Fowler, B. Lebeau, S. Mann, Chem. Commun. (1998) 1825-1826.

[16] R.P. Bagwe, L.R. Hilliard, W.H. Tan, Langmuir 22 (2006) 4357-4362.

[17] J.E. Smith, L. Wang, W.T. Tan, Tr. Trends Anal. Chem. 25 (2006) 848-855.

[18] X.C. Zhou, J.Z. Zhou, Anal. Chem. 76 (2004) 5302-5312.

[19] S. Santra, B. Liesenfeld, C. Bertolino, D. Dutta, Z.H. Cao, W.H. Tan, B.M. Moudgil, R.A. Mericle, J. Lumin. 117 (2006) 75-82.

[20] F. Gouanve, T. Schuster, E. Allard, R. Meallet-Renault, C. Larpent, Adv. Funct. Mater. 17 (2007) 2746-2756.

[21] O. Stranik, R. Nooney, C. McDonagh, B.D. MacCraith, Plasmonics 2 (2007) 1522.

[22] M. Qhobosheane, S. Santra, P. Zhang, W.H. Tan, Analyst 126 (2001) 1274-1278.

[23] S. Santra, D. Dutta, G.A. Walter, B.M. Moudgil,Tech. Cancer Res. Treat. 4 (2005) 593-602.

[24] S.R. Corrie, G.A. Lawrie, M. Trau, Langmuir 22 (2006) 2731-2737.

[25] W. Lian, S.A. Litherland, H. Badrane, W.H. Tan, D.H. Wu, H.V. Baker, P.A. Gulig, D.V. Lim, S.G. Jin, Anal. Biochem. 334 (2004) 135-144.

[26] K. Borchers, A. Weber, H. Brunner, G.E.M. Tovar, Microstructured layers of spherical biofunctional core-shell nanoparticles provide enlarged reactive surfaces for protein microarrays, Bad Heiligenstadt, GERMANY, 2004, pp. 738-746. 
[27] P.K. Ajikumar, J. Kiat, Y.C. Tang, J.Y. Lee, G. Stephanopoulos, H.P. Too, Langmuir 23 (2007) 5670-5677.

[28] R. Benters, C.M. Niemeyer, D. Wohrle, Chem. Biochem 2 (2001) 686-694.

[29] T. Bocking, E.L.S. Wong, M. James, J.A. Watson, C.L. Brown, T.C. Chilcott, K.D. Barrow, H.G.L. Coster, Thin Solid Films 515 (2006) 1857-1863.

[30] D.I. Rozkiewicz, W. Brugman, R.M. Kerkhoven, B.J. Ravoo, D.N. Reinhoudt, Dendrimer-mediated transfer printing of DNA and RNA microarrays, J. Am. Chem. Soc. 129 (2007) 11593-11599.

[31] A.E. Beezer, A.S.H. King, I.K. Martin, J.C. Mitchel, L.J. Twyman, C.F. Wain, Tetrahedron 59 (2003) 3873-3880.

[32] D. Shcharbin, M. Janicka, M. Wasiak, B. Palecz, M. Przybyszewska, M. Zaborski, M. Bryszewska, Biochim. Biophys. Acta, Proteins and Proteomics 1774 (2007) 946-951.

[33] A.E. Beezer, A.S.H. King, I.K. Martin, J.C. Mitchell, L.J. Twyman, C.F. Wain, Tetrahedron 59 (2003) 3873-3880.

[34] I.J. Majoros, A. Myc, T. Thomas, C.B. Mehta, J.R. Baker, Biomacromolecules 7 (2006) $572-579$.

[35] I.J. Majoros, J.R.B. Jr., Dendrimer-based Nanomedicine, Singapore, 2008.

[36] E. Buhleier, W. Wehner, F. Vogtle, Synthesis-Stuttgart (1978) 155-158.

[37] A. Hucknall, S. Rangarajan, A. Chilkoti, Adv. Mater. 21 (2009) 2441-2446.

[38] J.W.A. Findlay, W.C. Smith, J.W. Lee, G.D. Nordblom, I. Das, B.S. DeSilva, M.N. Khan, R.R. Bowsher, J. Pharm. Biomed. Anal. 21 (2000) 1249-1273.

[39] P.B. Rahul, L.R. Hillard, W. Tan, Langmuir 22 (2006) 4357-4362.

[40] L.R. Hilliard, X.J. Zhao, W.H. Tan, Anal. Chim. Acta 470 (2002) 51-56.

[41] Pierce Technical Note 0650.6, www.thermo.com/pierce

[42] K. Nam, T. Kimura, A. Kishida, Macromol. Biosci. 8 (2007), 32-37

[43] L.A. Cantarero, J.E. Butler, J.W. Osborne, Anal. Biochem. 105 (1980) 375-382. 


\section{Figures}

Figure 1 Monovalent and multivalent cross-linkers used in this work. Inset in the cartoon view of dendrimer depicting the activation of the carboxylic acids and the two competing reactions occurring on the carbonyl group.

Figure 2 A TEM micrograph of dye doped silica NPs approximately $71+/-4 \mathrm{~nm}$ in total diameter.

Figure 3 Dynamic Light Scattering data showing the rate of NP aggregation (A) as effect of the corresponding linker attached to its surface; and (B) change in zeta potential as a function of the change on the surface of the NPs

Figure 4 Normalized fluorescence intensity measured after immobilization of Green Fluorescent Protein onto the NP surface. Optimal conditions were determined by (A) - varying the stoichiometric ratio between EDC / NHS for the activation of G2 dendrimer for 30 minutes, followed by: (B) - variations in the activation time of the G2 dendrimer at the optimum EDC/NHS ratio (4:1).

Figure 5 Normalized fluorescence intensity measured after immobilization of Green Fluorescent Protein on the NP surface modified with sulfo-SMCC and the G2 dendrimer. In these reactions, both the amount of the NIR-NP and the cross linker were kept constant, and the concentration of the GFP was 1.5 or 3.0 equivalents respectively. 1 equivalent represents an amount of protein (in $\mu \mathrm{g}$ ) that is necessary for a surface saturation of $1 \mathrm{mg}$ of the nanoparticles. This value was calculated according to the formula in ref. 36.

Figure 6 Fluoresence linked immunosorbent assays for the detection of $\mathrm{hIgG}$ using monovalent and multivalent bilinkers. 
$0 \approx \sim>0$

Glutaraldehyde (glut)

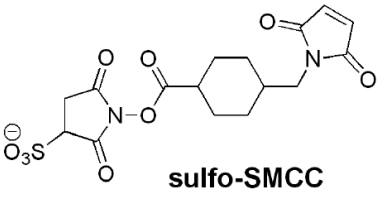

MULTIVALENT LINKERS

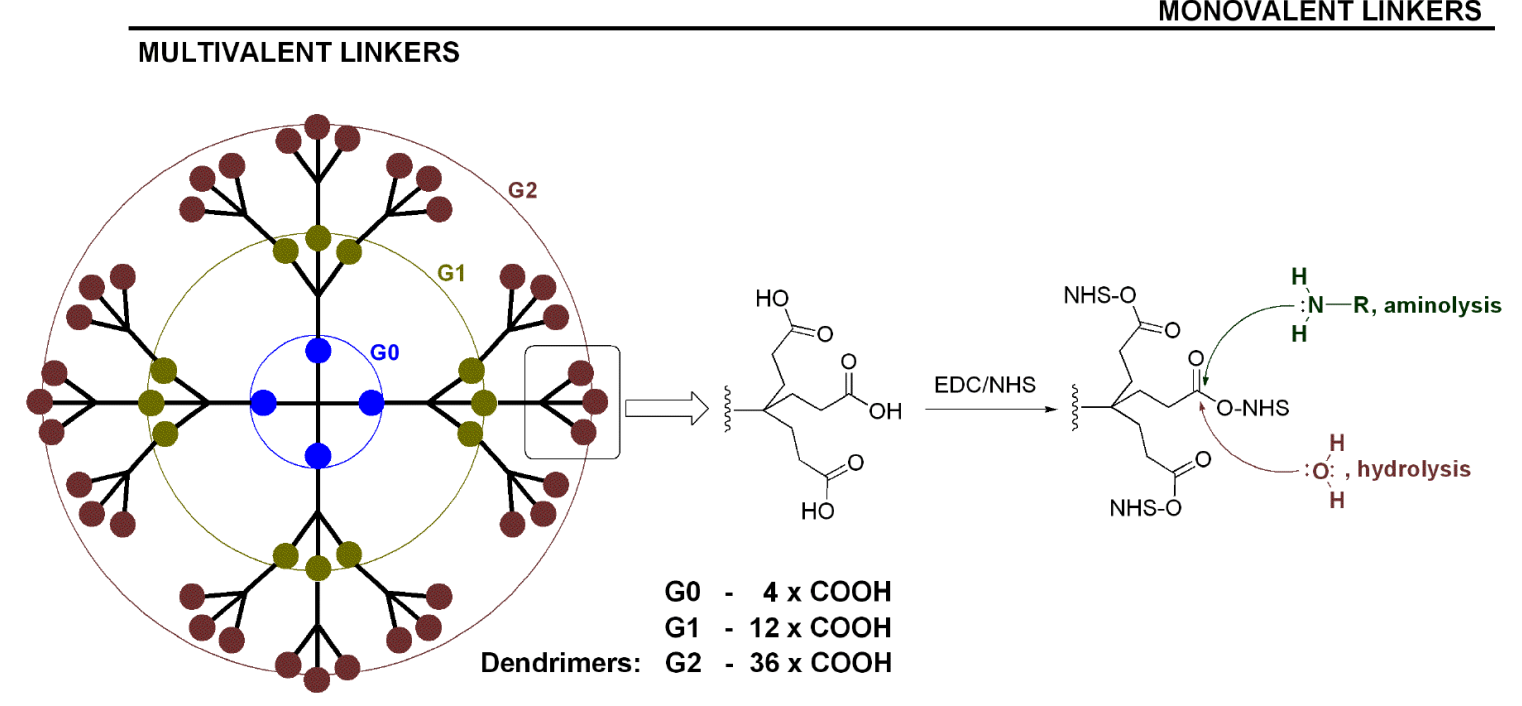

Figure 1

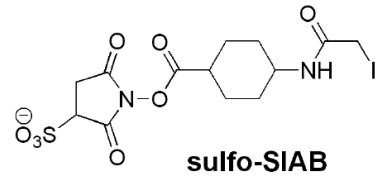

MONOVALENT LINKERS 


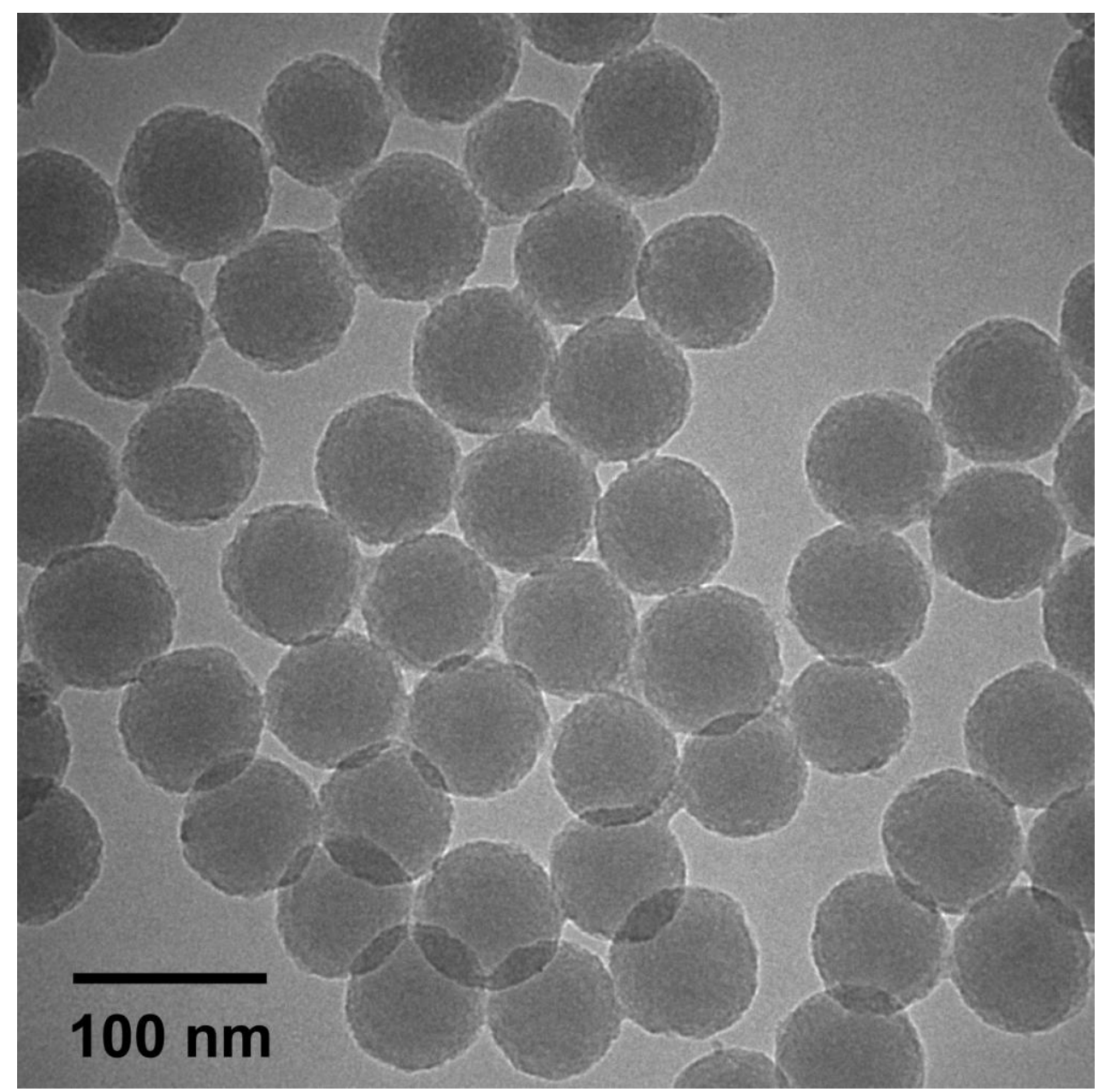

Figure 2
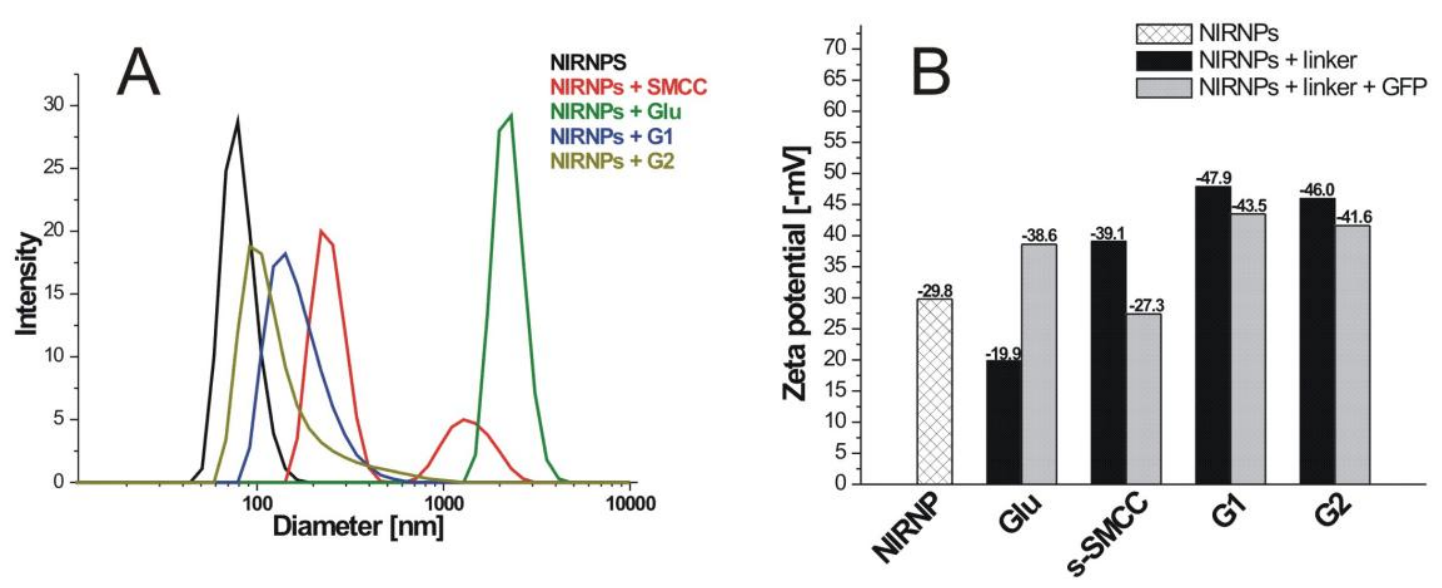

Figure 3 

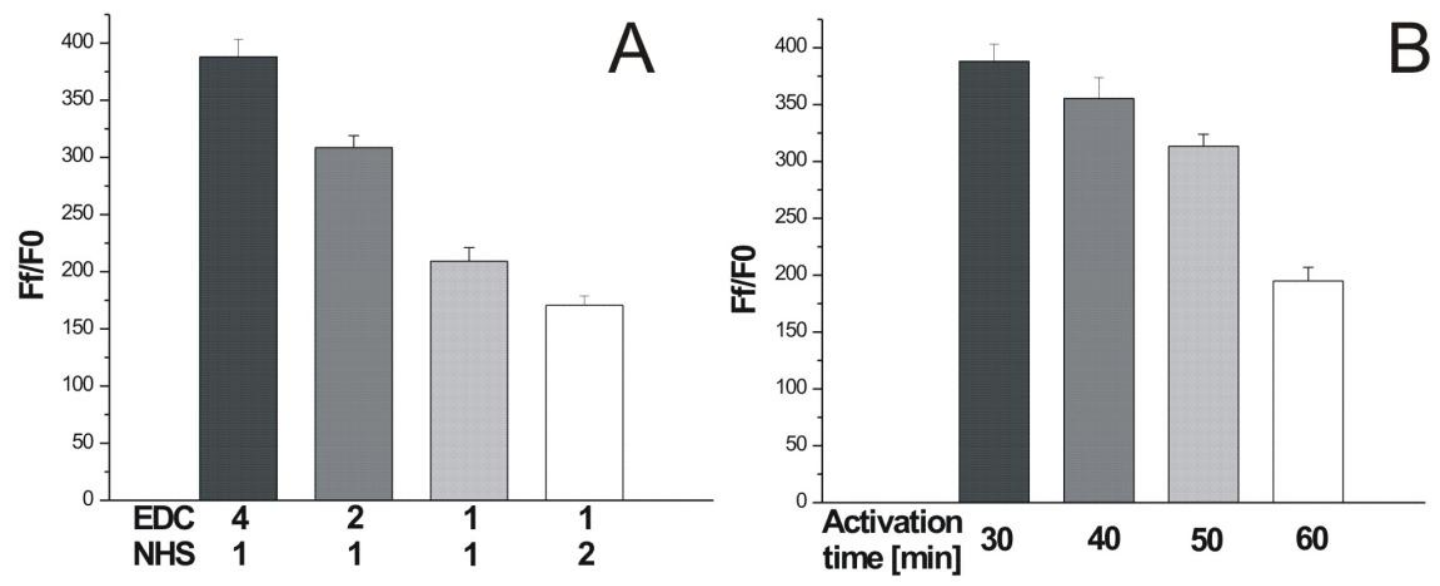

Figure 4

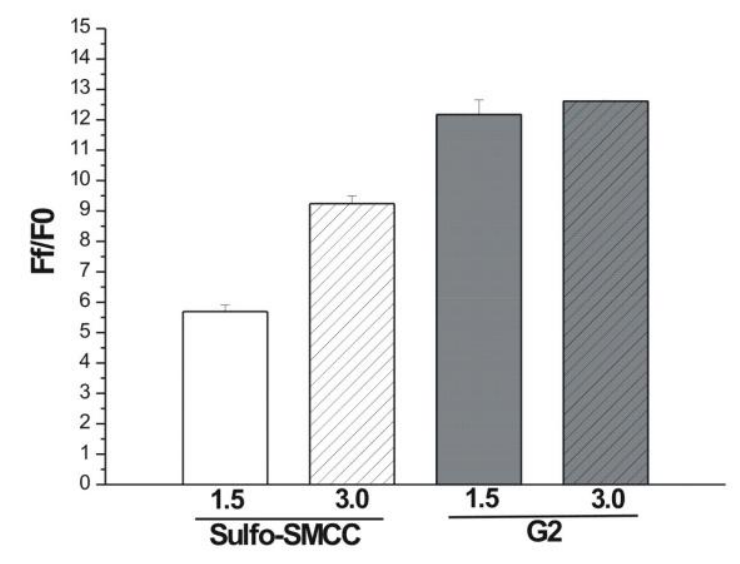

Figure 5 


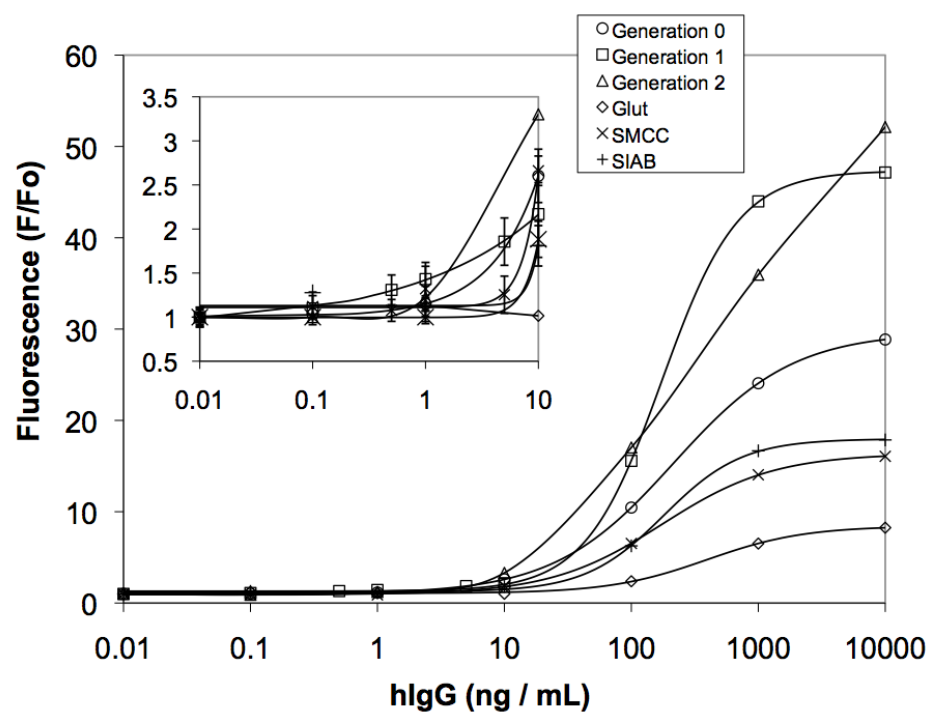

Figure 6 


\section{Tables}

\begin{tabular}{|l|c|c|c|c|c|c|}
\cline { 2 - 7 } \multicolumn{1}{c|}{} & \multicolumn{3}{c|}{ Multi-valent linkers } & \multicolumn{3}{c|}{ monovalent Linkers } \\
\cline { 2 - 7 } \multicolumn{1}{c|}{} & \multicolumn{3}{c|}{ Dendrimer generations } & Homo- & \multicolumn{2}{c|}{ Hetero- } \\
\cline { 2 - 7 } \multicolumn{1}{c|}{} & 0 & 1 & 2 & Glut & SMCC & SIAB \\
\hline Fmax/Fo & 29.67 & 47.34 & 52.13 & 8.43 & 16.37 & 17.98 \\
\hline CV\% (no analyte) & 8.19 & 10.95 & 14.06 & 17.90 & 11.34 & 4.40 \\
\hline X(LOD (ng/mL) & 1.66 & 0.31 & 2.67 & 41.96 & 7.55 & 7.09 \\
\hline
\end{tabular}

Table 1 The total fluorescence signal over background fluorescence signal, $F / F o$ combined with coefficient of variance, and LOD results for each linker in the fluorescence linked immunosorbent assay for detection of human $\operatorname{IgG}$ 